\title{
Mach Number Effects on Conical Surface Features of Swept Shock-Wave/Boundary-Layer Interactions
}

\author{
F. K. Lu* and G. S. Settles† \\ Pennsylvania State University, University Park, Pennsylvania \\ and \\ C. C. Horstman $\ddagger$ \\ NASA Ames Research Center, Moffett Field, California
}

\begin{abstract}
An experiment and some computational comparisons are described concerning the shock-wave turbulent boundary-layer interactions generated by sharp fins, with emphasis on Mach number effects. The Mach number range is from 2.5 to 4, and the unit Reynolds number is from 50 to $80 \times 10^{6} / \mathrm{m}$. Fin angles are varied from 4 to $22 \mathrm{deg}$. Surface flow patterns are obtained using a color surface-flow visualization technique. The results show that the "upstream-influence response" in the conical far-field region is a function of the freestream Mach number and the shock strength. A new interpretation of the behavior of the upstream influence with changes of the inviscid shock angle is given. Variations of the interaction response with Mach number are observed to be due almost entirely to the shock strength, and not to compressibility effects on turbulence, over the Mach number range considered. The overall agreement between the experimental and computed interactions is good, though the computation underpredicts the upstream influence line somewhat for the stronger interactions.
\end{abstract}

\section{Nomenclature}

$a, b \quad=$ constants in power of $R e_{\delta}$ in Reynolds number scaling law, Eq. (1a)

$c_{f} \quad=$ incoming boundary-layer skin-friction coefficient

$k_{1}, k_{2}=$ constants in $\beta_{U}$ correlation, Eq. (3)

$\left(l_{s}, l_{n}\right)=$ orthonormal coordinates based on inviscid shockwave trace on test surface, (Fig. 2b), $\mathrm{mm}$ (in.)

$L_{n} \quad=$ nondimensional distance measured normal to inviscid shock-wave trace on the test surface, $=\left(l_{n} / \delta\right) \operatorname{Re}_{\delta}^{a}$

$L_{s} \quad=$ nondimensional distance measured along inviscid shock-wave trace on the test surface, $=\left(l_{s} / \delta\right) R e_{\delta}^{b}$

$M_{n} \quad=$ Mach number normal to inviscid shock-wave trace on test surface, $=M_{\infty} \sin \beta_{o}$

$M_{\infty} \quad$ incoming freestream Mach number

$M_{2} \quad$ = freestream Mach number downstream of shock

$p_{0} \quad=$ stagnation pressure of incoming stream, $\mathrm{MPa}$ (psia)

$p_{1} \quad$ = incoming freestream static pressure, MPa (psia)

$p_{2} \quad=$ freestream static pressure downstream of shock, MPa (psia)

$p_{r} \quad=$ inviscid pressure ratio across shock, $=p_{2} / p_{1}$

$r \quad=$ distance measured from the virtual origin, (Fig. 2b), mm (in.)

$R e=$ unit Reynolds number, $\mathrm{m}^{-1}(/ \mathrm{ft})$

$R e_{\delta}=$ Reynolds number based on the local, undisturbed boundary-layer thickness

$T_{w} \quad=$ wall temperature, $\mathrm{K}\left({ }^{\circ} \mathrm{R}\right)$

$T_{o} \quad=$ freestream stagnation temperature, $\mathrm{K}\left({ }^{\circ} \mathrm{R}\right)$

Presented as Paper 87-1365 at the AIAA 19th Fluid Dynamics, Plasma Dynamics, and Lasers Conference, Honolulu, HI, June 8-10, 1987; received Sept. 28, 1987; revision received Jan. 23, 1989. Copyright (C) 1987 American Institute of Aeronautics and Astronautics, Inc. All rights reserved.

${ }^{*}$ Graduate Research Assistant, Mechanical Engineering Department; presently Assistant Professor, Aerospace Engineering Department, The University of Texas, Arlington, TX. Member AIAA.

†Professor, Mechanical Engineering Department and Director, Gas Dynamics Laboratory. Associate Fellow AIAA.

$\ddagger$ Assistant Branch Chief, Experimental Fluid Dynamics Branch. Associate Fellow AIAA. $\alpha \quad=$ angle made by fin with respect to the incoming freestream direction, deg

$\beta \quad=$ angle made by surface-flow features with incoming freestream direction, deg

$\beta_{0} \quad=$ angle made by inviscid shock-wave trace on test surface with incoming freestream direction, deg

$\Delta \beta_{U}=\left(\beta_{\mathrm{U}}-\mu_{\infty}\right), \mathrm{deg}$

$\Delta \beta_{o}={ }^{*}\left(\beta_{o}-\mu_{\infty}\right)$, deg

$\delta \quad=$ local, undisturbed boundary-layer thickness, mm (in.)

$\delta^{*} \quad=$ local, undisturbed boundary-layer displacement thickness, mm (in.)

$\theta \quad=$ local, undisturbed boundary-layer momentum thickness, mm (in.)

$\kappa_{1}, \kappa_{2}=$ constants in scaling of $\Delta \beta_{U}$ with $\Delta \beta_{o}$, Eq. (12)

$\lambda=$ fin leading-edge sweepback angle, deg

$\mu_{\infty}=$ Mach angle of the incoming freestream, $\mathrm{deg},=\arcsin \left(1 / M_{\infty}\right)$

$\Pi=$ incoming boundary-layer wake-strength parameter

Subscripts

$i \quad=$ inception

$U \quad=$ upstream influence

max $=$ highest fin angle-of-attack

$\infty \quad=$ incoming freestream conditions

\section{Introduction}

S HOCK-WAVE boundary-layer interactions continue to be studied as both basic and practical problems of high-speed fluid dynamics. Although research on such problems in the past concerned mainly two-dimensional interactions, the last decade or so has seen progress on the problems of three-dimensional interactions as well. This topic was recently reviewed by Settles and Dolling, ${ }^{1}$ who broadly divided three-dimensional shock-wave/boundary-layer interactions into semi-infinite and non-semi-infinite classes. Within the semi-infinite class is a subclass of dimensionless interactions of which a shock generator mounted perpendicularly to a flat surface forms an archetypal family (Fig. 1). This geometry is also commonly called called a "fin," with obvious connotations. 


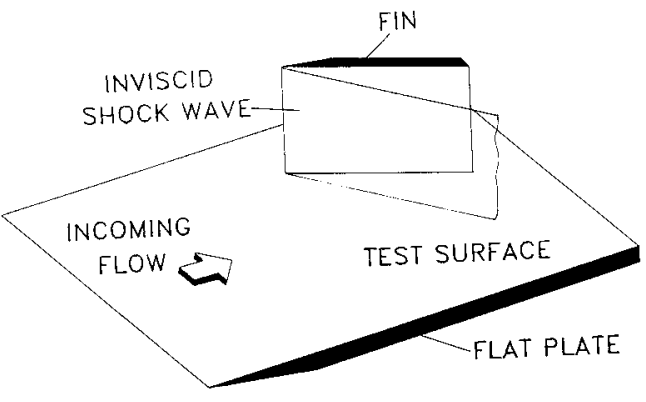

TEST GEOMETRY

Fig. 1 Test model

\section{Brief Review of Related Studies}

For practical reasons, past experimental studies of fin-generated interactions have concentrated on those involving turbulent boundary layers. A useful basic technique in these studies is surface-flow visualization; an example of such a surface-flow pattern is shown in Fig. 2a; the key features in Fig. $2 \mathrm{a}$ are identified in Fig. 2b. The fin, placed at an angle $\alpha$ to the incoming stream, generates an inviscid shock wave that impinges on the test surface at an angle $\beta_{o}$. The upstream influence line is the location of the onset of the interaction. The "separation line," if it occurs, is characterized by a convergence of the surface streaks. This is believed to indicate that an open boundary-layer separation exists. Associated with the separation line is an attachment line. Within the separated region bounded by the separation and attachment lines is a strong swept vortical flow. Separation occurs only when the shock provoking the interaction is sufficiently strong. Early studies showed that the incipient separation condition occurs when $M_{n} \approx 1.2$ or, equivalently, $p_{r} \approx 1.5$. For the strongest interactions a "secondary separation" line is also observed, although not much is known about this phenomenon.

Settles and $\mathrm{Lu}^{2}$ found that the surface-flow pattern in fingenerated interactions revealed an initial "inception region", near the fin leading edge and a far-field region further away (Fig. 2b). The surface features in the far field appear to radiate from a "virtual origin," thus displaying conical symmetry. This conical symmetry was also found analytically by Inger $^{3}$ and was alluded to by Hayes ${ }^{4}$ and Zubin and Ostapenko in their experimental studies. Inger found that although the boundary-layer growth cannot be conical, the conical nature of the inviscid freestream nonetheless forces the interaction to tend toward a conical form in the far field. From the experimental studies, therefore, an appropriate coordinate system to qualify the surface features of such interactions (at least for the region between the upstream influence line and the inviscid shock-wave trace) is a polar coordinate system $(r, \beta)$ centered at the virtual origin, as illustrated in Fig. $2 b$.

An excellent approximation to the polar coordinate system described is an orthonormal coordinate system $\left(l_{s}, l_{n}\right)$, also shown in Fig. 2 b. In this coordinate frame, $l_{s}$ is distance measured along the inviscid shock-wave trace on the test surface and $l_{n}$ is distance measured normal to $l_{s}$.

Dolling and Bogdonoff ${ }^{6}$ and Settles and $\mathrm{Lu}^{2}$ found that the upstream influence line in a Mach 3 fin-generated interaction obeys the following Reynolds number scaling law:

$$
\left[\frac{l_{n}}{\delta} R e_{\delta}^{a}\right]_{U} M_{n}=f\left(\left[\frac{l_{s}}{\delta} R e_{\delta}^{b}\right]_{U}\right)
$$

or

$$
\frac{\left[L_{n}\right]_{U}}{M_{n}}=f\left(\left[L_{s}\right]_{U}\right)
$$

In Eq. (1a), the empirically derived constants $a$ and $b$ were both found to be equal to $1 / 3$. Equation (1b) was further found

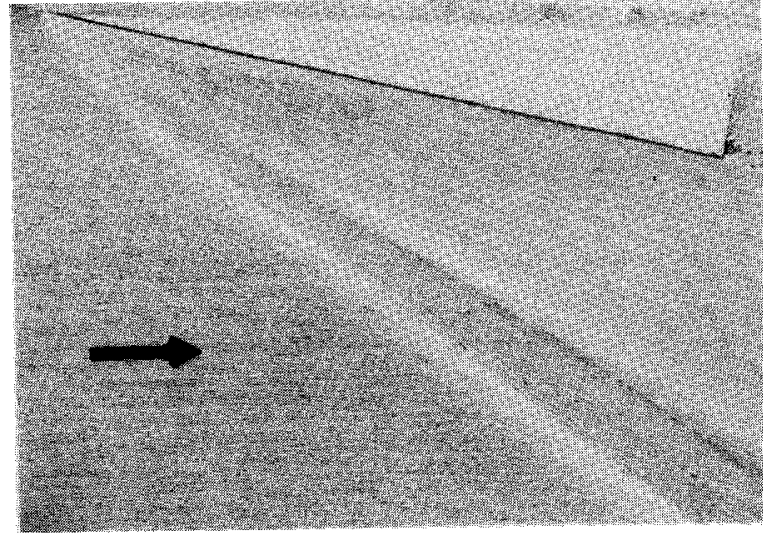

a)

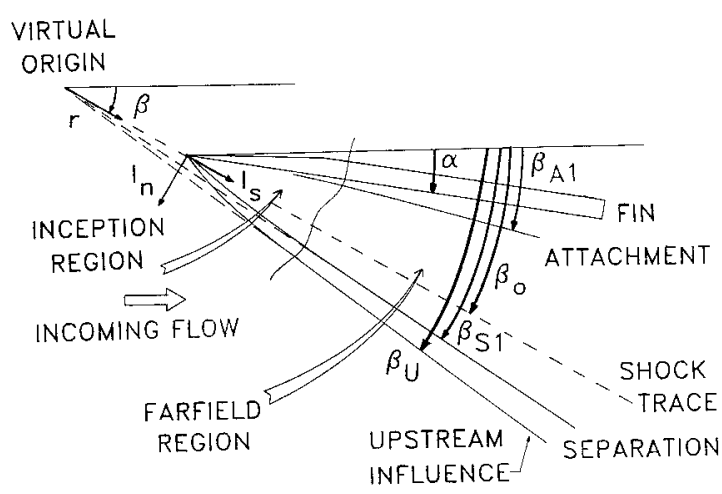

b)

Fig. 2 Surface-flow visualization results: a) example at $M_{\infty}=\mathbf{2 . 9 5}$, $\alpha=15 \mathrm{deg}$; b) sketch showing key surface features.

to hold for fins with swept leading edges. Subsequently, Settles ${ }^{7}$ found that the nondimensional inception length to conical symmetry for Mach 3 fin-generated interactions, $L_{s_{i}}$, is related to $\beta_{o}$ by

$$
L_{s} \approx 1130 \cot \beta_{o}
$$

Thus, Settles ${ }^{7}$ identified the dependence of $l_{s_{i}}$, on both viscous and inviscid parameters.

There have been attempts to understand the behavior of the observed surface-flow-pattern features in the conically symmetric region. ${ }^{2,8-10}$ Basically, these studies found that the salient indicator of interaction "response," the upstream-influence angle $\beta_{U}$, could be expressed as

$$
\beta_{U}=k_{1} \beta_{o}-k_{2}
$$

where $k_{1}=1.74$ and is independent of Mach number and $k_{2}$ is a decreasing function of Mach number. ${ }^{10} \mathrm{~A}$ more physical correlation, based in part on Eq. (1b), was given by Settles and $\mathrm{Lu}^{2}$ for Mach 3 interactions as

$$
\left(\beta_{U}-\beta_{o}\right)=\arctan \left(0.26 \sin \beta_{o}\right)
$$

The linearity of $\beta_{U}$ with $\beta_{o}$, expressed by Eq. (3) or (4), appears valid for fins with swept as well as unswept leading edges over a limited range of fin angle $\alpha$.

The study by Settles and $\mathrm{Lu}^{2}$ also found that other key surface feature angles, such as $\beta_{S 1}, \beta_{A 1}$, and $B_{S 2}$ (see Fig. 2b), increase with shock strength. In addition, just as for $\beta_{U}$, these angles, obtained with upright fins, correlated with those obtained with fins having swept leading edges. Settles and $\mathrm{Lu}$ concluded that planar or weakly curved inviscid shocks of the same $\beta_{o}$ produce essentially equivalent interactions. This is 
because the effect of the shock is a local one in the immediate vicinity of the boundary layer. In this local region, the inviscid shocks have large radii of curvature, regardless of fin leadingedge sweepback.

Other than experimental studies, numerical predictions of the interaction using approximations to the Navier-Stokes equations were recently performed by several investigators, including Horstman. ${ }^{11}$ Horstman's results revealed that a flat vortex exists when the boundary layer is separated, a fact that agreed with experimental observations. ${ }^{2,8}$ The computed skinfriction lines and surface pressure distributions also showed that the far-field surface features are conically symmetric. Some quantitative disagreement between the computed and measured interaction scales has been found, however, especially for stronger interactions. This issue will also be addressed in the present study.

\section{Present Study}

As noted, fin-generated interactions have been relatively well-studied at Mach 3 . However, no comprehensive study has been done through a wide Mach number range. The effect of Mach number on the interaction is, therefore, an important unknown. Previous studies, ranging from Mach 2-11 (such as Refs. 4 and 12-14), are generally inadequate for this purpose in that insufficiently detailed measurements were reported. This is especially so of the upstream influence, even though its determination is important in both practical and theoretical terms.

In addition, most fin interaction studies were done at one or two Mach numbers only and were thus unable to reveal Mach number trends. Some studies were also contaminated by windtunnel sidewall interference or were carried out within the inception zone. Data for such experiments obviously cannot be correlated using the otherwise powerful concept of conical symmetry.

A new experimental program was thus begun at the Pennsylvania State University Gas Dynamics Laboratory to explore fin-generated turbulent interactions through a Mach number range of 2.5-4. Concurrently, Navier-Stokes solutions were performed at NASA Ames Research Center to judge the ability of the state-of-the-art computational techniques to predict such flows. The goals of this joint program are 1) to gain a physical understanding of the effect of Mach number on these interactions in the supersonic range, 2) to provide "benchmark" data for code validation purposes, and 3) to test the performance of current computational fluid dynamics methods in predicting these complex flows and guide the way toward computational improvements. This paper reports the first phase of this research program, which concentrates on the far-field upstream influence of fin interactions as revealed by surface-flow visualization.

\section{Experimental Methods}

\section{Wind Tunnel and Test Models}

The experimental study was done in the Supersonic Wind Tunnel Facility (SWT) of the Pennsylvania State University Gas Dynamics Laboratory. This wind tunnel is a blowdown type with a nominal Mach number range from 1.5-4. The variable Mach number capability is achieved by way of an asymmetric sliding-block nozzle. The SWT has a test section $150 \mathrm{~mm}$ (6 in.) wide, $165 \mathrm{~mm}$ (6.5 in.) high, and $610 \mathrm{~mm}(24$ in.) long. (Further description of the wind tunnel and the experiments can be found in Ref. 15.)

For the present tests, a flat plate, $500 \mathrm{~mm}(19.5 \mathrm{in}$.$) long,$ spanning the tunnel, was mounted in the test section to provide the interaction test surface. An equilibrium turbulent boundary layer formed on this plate with natural transition typically occurring within $1 \mathrm{~cm}$ of the leading edge. A fin model with a 10 deg sharp leading edge was placed with its tip $216 \mathrm{~mm}$ ( $8.5 \mathrm{in}$.) from the plate leading edge and $26.2 \mathrm{~mm}$ (1.03 in.) from the tunnel sidewall. The fin was $100 \mathrm{~mm}$ (4 in.) high, $127 \mathrm{~mm}$ ( 5 in.) long, and $6.35 \mathrm{~mm}(0.25$ in.) thick. The fin height of about $30 \delta$ was, therefore, sufficient to insure that the interaction was a semi-infinite one. ${ }^{1,16}$ The length of the fin was chosen to provide the maximum interaction extent while allowing sufficiently large angles of attack to be obtained without stalling the wind tunnel.

The fin was held tightly onto the flat plate by a fin-injection mechanism mounted on the tunnel sidewall. A rubber seal at the bottom of the fin insured that no leakage under the fin occurred during the tests. The fin-injection mechanism pneumatically injected the fin to a present angle of attack $\alpha$ once test conditions were established. This was necessary only for tests with $\alpha$ larger than approximately $14 \mathrm{deg}$. At lower angles $\alpha$ was fixed before the run. In the experiments $\alpha$ ranged from 4 to $22 \mathrm{deg}$, the largest value being limited by tunnel stalling. The fin angle was determined to 0.1-deg accuracy using a machinist's protractor.

\section{Test Conditions}

The Mach numbers of the experiments were 2.47, 2.95, 3.44, and 3.95. The incoming freestream conditions at these Mach numbers are summarized in Table 1 , where the \pm percentages indicate repeatability in terms of the ensemble-average standard deviation for all tests at a given Mach number. Since the wind tunnel is a blowdown type, the stagnation temperature decreased somewhat during a run. Typically, for a run of about $20 \mathrm{~s}$, the stagnation temperature $T_{o}$ dropped from $300 \mathrm{~K}$ $\left(540^{\circ} \mathrm{R}\right)$ to $290 \mathrm{~K}\left(520^{\circ} \mathrm{R}\right)$. The nominal freestream unit Reynolds number $R e$ was held relatively constant throughout the Mach number range at $50-80 \times 10^{6} \mathrm{~m}^{-1}\left(15-24 \times 10^{6} / \mathrm{ft}\right)$. In order to achieve this, the tunnel stagnation pressure $p_{o}$ had to be increased with Mach number, as can be seen from Table 1.

Figure 3 is an example of an undisturbed plate-centerline boundary-layer velocity profile in law-of-the-wall coordinates. The figure also shows the Sun-Childs ${ }^{17}$ wall-wake curvefit to the data. Detailed surveys along the flat plate centerline and 38 $\mathrm{mm}(1.5 \mathrm{in}$.) to each side showed that the boundary layers were

Table 1 Incoming freestream conditions

\begin{tabular}{cccc}
\hline$M_{\infty}$ & $\begin{array}{c}p_{o}, \mathrm{MPa} \\
(\mathrm{psia})\end{array}$ & $\begin{array}{c}T_{o}, \mathrm{~K} \\
\left({ }^{\circ} \mathrm{R}\right)\end{array}$ & $\begin{array}{c}R e \times 10^{-6}, \mathrm{~m}^{-1} \\
(/ \mathrm{ft})\end{array}$ \\
\hline $2.47 \pm 0.1 \%$ & $\begin{array}{c}0.54 \pm 2.0 \% \\
(78)\end{array}$ & $\begin{array}{c}295+0.9 \% \\
(531)\end{array}$ & $\begin{array}{c}53.8 \pm 0.9 \% \\
(16.3)\end{array}$ \\
$2.95 \pm 0.3 \%$ & $\begin{array}{c}0.76 \pm 2.7 \% \\
(110)\end{array}$ & $\begin{array}{c}295 \pm 0.9 \% \\
(531)\end{array}$ & $\begin{array}{c}58.9 \pm 1.9 \% \\
(17.8)\end{array}$ \\
$3.44 \pm 0.2 \%$ & $\begin{array}{c}1.03 \pm 3.0 \% \\
(150)\end{array}$ & $295 \pm 0.8 \%$ & $64.0 \pm 1.7 \%$ \\
$3.95 \pm 0.4 \%$ & $1.58 \pm 5.0 \%$ & $295 \pm 1.3 \%$ & $(19.4)$ \\
& $(230)$ & $(531)$ & $75.8 \pm 1.7 \%$ \\
& & & $(23.0)$ \\
\hline
\end{tabular}

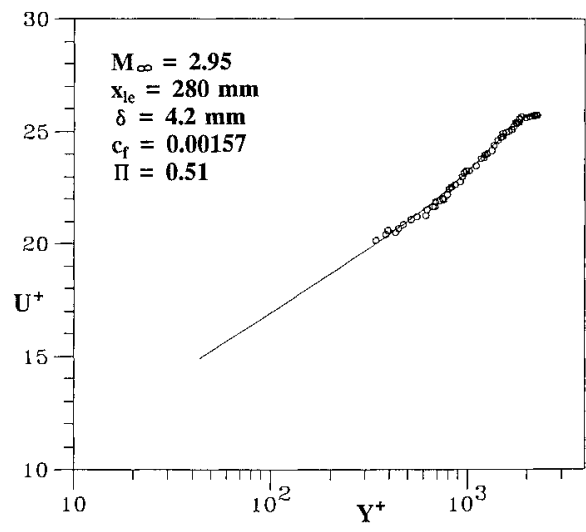

Fig. 3 Undisturbed boundary-layer profile. 
Table 2 Incoming boundary-layer parameters

\begin{tabular}{cccc}
\hline$M_{\infty}$ & 2.47 & 2.95 & 3.44 \\
\hline$\delta, \mathrm{mm}$ & $3.60 \pm 0.14$ & $3.63 \pm 0.04$ & $3.24 \pm 0.06$ \\
$\delta^{*}, \mathrm{~mm}$ & $0.82 \pm 0.02$ & $0.93 \pm 0.11$ & $0.92 \pm 0.05$ \\
$\theta, \mathrm{mm}$ & $0.22 \pm 0.004$ & $0.19 \pm 0.02$ & $0.15 \pm 0.01$ \\
$c_{f} \times 10^{3}$ & $1.78 \pm 0.07$ & $1.64 \pm 0.12$ & $1.51 \pm 0.40$ \\
II & $0.63 \pm 0.25$ & $0.58 \pm 0.22$ & $0.51 \pm 0.06$ \\
\hline
\end{tabular}

two-dimensional. The boundary layers were turbulent and in equilibrium at the test region and were at approximately adiabatic conditions. The incoming boundary-layer parameters, measured at $x=0.229 \mathrm{~m}$ ( 9 in.) downstream of the flat plate leading edge, are given in Table 2 . Note that these tabulated parameters were obtained from wall-wake curvefits to the data and that no data are shown for Mach 3.95 since boundarylayer surveys have not been completed at that Mach number. A more detailed discussion of the test boundary layers is available in Ref. 15.

\section{Experimental Techniques}

Temperature data from thermocouples and pressure data from pressure transducers were digitized and stored in a microcomputer. The computer was used to display tunnel conditions during a test as well as to analyze data. Further data analysis was performed on a mainframe computer.

The surface-flow features were visualized using a kerosenelampblack technique. ${ }^{18}$ In addition, an improvement over this well-tried technique used powered colored chalk as the pigment material in a kerosene carrier. The colors helped to distinguish some of the important features of the surface flow, particularly the separation line. Spatial data obtained from full-size undistorted images of the surface pattern (preserved on matte acetate tape) were accurate to $0.5 \mathrm{~mm}(0.02 \mathrm{in}$.); these data were also digitized and stored in a microcomputer for analysis. Angular data were accurate to $\pm 0.5 \mathrm{deg}$ for stronger interactions, but this accuracy was found to be as poor as \pm 3 deg for weak interactions due to the difficulty in discerning weak surface-flow features.

The surface-flow visualization technique reveals only the average skin-friction lines of the interaction. It is recognized that such turbulent interactions fluctuate locally over a broad frequency range, ${ }^{1}$ though the fluctuation scale is observed to be much smaller than the global scale of the interaction. In particular, recent work by Gramann and Dolling ${ }^{19}$ indicates that the zone between the surface-flow upstream influence and "separation" lines is actually characterized by intermittent flow separation. Since the present goal concerns the overall interaction scaling rather than its unsteadiness, the mean data shown here do not conflict with this view of the interaction as an unsteady process.

\section{Computations}

The computations, using the MacCormack explicit, secondorder, predictor-corrector, finite-volume method, solve the Reynolds-averaged Navier-Stokes equations with a two-equation $k-\epsilon$ turbulence model. ${ }^{11}$ A detailed description of the boundary conditions imposed around the borders of the computational domain can be found in Reference 20 . Briefly, the freestream and boundary-layer conditions at the upstream boundary of the computational domain were chosen to match those of the experiment. No-slip, adiabatic-wall conditions were applied at all solid surfaces. On the flow boundary opposite the fin, spanwise gradients of flow variables were set to zero, while on the upper flow boundary the normal components of flow variables were set to zero. The shock wave passed through the downstream boundary of the domain, where streamwise gradients of the flow variables were set to zero.

Computations were made at Mach 2 and 4 for interactions generated by $\alpha=5,14$, and 20 deg fine. Furthermore, for the

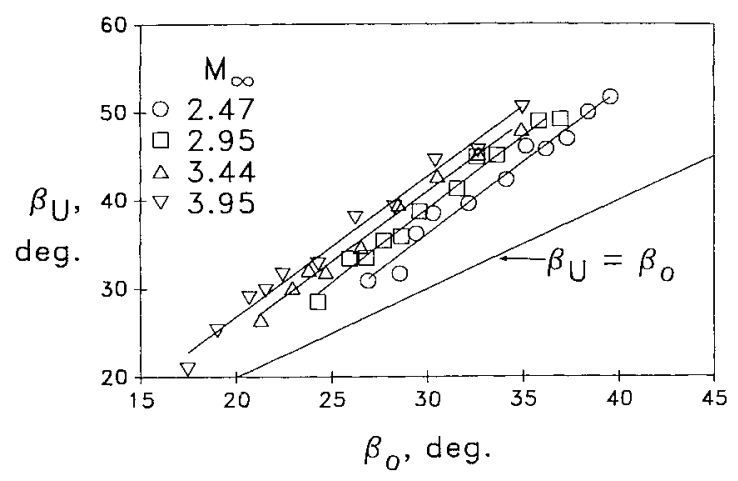

Fig. 4 Upstream influence (raw data).

first time, computations of a swept leading-edge fin-generated interaction were made. Two cases at Mach 3 were considered, namely, interactions generated by an $\alpha=15 \mathrm{deg}, \lambda=20 \mathrm{deg}$ fin and an $\alpha=15 \mathrm{deg}, \lambda=40 \mathrm{deg}$ fin.

\section{Upstream Influence}

\section{Discussion of Experimental Results}

As a first step in the data analysis, the raw $\beta_{U}$ data are plotted in Fig. 4 against $\beta_{o}$. The line $\beta_{U}=\beta_{\mathrm{o}}$ in the figure amounts to a lower bound for vanishingly weak interactions. At the other extreme, the largest value of $\beta_{U}$ for a given $M_{\infty}$, by extrapolation of data, is presumed to correspond to the largest $\alpha$ before inviscid shock detachment, $\alpha_{\max }$. For $M_{\infty}>2$, $\beta_{o}$ is almost constant ( $\approx 65-68 \mathrm{deg}$ ) for fins at the angle $\alpha_{\max }$. Therefore, the range of $\beta_{o}$ that can be generated by a variableangle fin widens as $M_{\infty}$ increases because of the decrease of $\mu_{\infty}$ rather than any increase of the maximum shock angle. The result is that $\beta_{U}$ also has a wider available range of values at higher $M_{\infty}$.

Figure 4 further shows that, at a given $\beta_{o}, \beta_{U}$ increases with $M_{\infty}$. To obtain the same $\beta_{o}$ requires that $\alpha$ increase with $M_{\infty}$. This, therefore, implies that $\beta_{U}$ in fact increases with the strength of the interaction, regardless of $M_{\infty}$. Conversely, for a given $\alpha, \beta_{U}$ decreases with increasing $M_{\infty}$. In other words, as $M_{\infty}$ increases for a given $\alpha$, the interaction extent (in angular terms) decreases. Furthermore, since the inviscid shock pressure ratio increases with $M_{\infty}$ for a constant $\alpha$, there will be larger interaction pressure gradients as $M_{\infty}$ increases because the pressure rise has to occur over a smaller angular extent. This explain why boundary-layer separation is observed to occur at lower $\alpha$ as $M_{\infty}$ increases, a fact that is well documented. ${ }^{12,14,21}$

\section{Dimensional Analysis}

In attempting to formulate a Mach number scaling law for $\beta_{U}$, it is appropriate to begin with dimensional analysis. The functional dependence of $\beta_{U}$ on the controlling parameters can be written as

$$
\beta_{U}=f_{U}\left(l_{s}, \delta, \operatorname{Re}_{\delta}, M_{\infty}, \beta_{o}\right)
$$

for given $T_{w} / T_{o}$ conditions. Note that the fin geometry, given by $\alpha$, is implicit in $\beta_{o}$ since the inviscid shock-wave trace depends on the fin geometry as well as on $M_{\infty}$. Equation (5) can be conveniently rewritten as

$$
\beta_{U}\left(L_{s}\right)=f_{U}\left(R e_{\delta}, M_{\infty}, \beta_{o}\right)
$$

Since the present study examines Mach number effects, $R e_{\delta}$ is held approximately constant so that its influence may be neglected. Additionally, in the conical far-field region where $L_{s}>L_{s}$, the explicit geometrical dependence can also be dropped since the effect of fin geometry is felt directly only in 
the inception zone. Equation (6) is, therefore, simplified to

$$
\beta_{U}\left(L_{s}>L_{s_{i}}\right)=f_{U}\left(M_{\infty}, \beta_{o}\right)
$$

For convenience, the argument of $\beta_{U}$ is omitted in the subsequent discussion.

For extremely weak interactions, when $\beta_{o} \rightarrow \mu_{\infty}$, is is postulated that $\beta_{U} \rightarrow \mu_{\infty}$ is a limiting condition on Eq. (7). (Experiments under such circumstances are scarce and difficult to perform and the results have large scatter.) Equation (7) is thus rewritten to reference both $\beta_{o}$ and $\beta_{U}$ properly to their limiting values:

$$
\left(\beta_{U}-\mu_{\infty}\right)=f_{U}\left[M_{\infty},\left(\beta_{o}-\mu_{\infty}\right)\right]
$$

or, simplifying the notation,

$$
\Delta \beta_{U}=f_{U}\left(M_{\infty}, \Delta \beta_{o}\right)
$$

where $\Delta \beta_{U}$ amounts to a reduced upstream influence response function while $\Delta \beta_{o}$ is a reduced shock-strength function.

\section{Physical Interpretation of Upstream Influence Sealing}

To understand the physics behind the functional relationship given by Eq. (8), it is necessary to begin by examining the classical oblique-shock relationship between $\beta_{o}, \alpha$, and $M_{\infty}$, which is a rather complicated one in general:

$$
\cot \alpha=\tan \beta_{o}\left[\frac{(\gamma+1) M_{\infty}^{2}}{2\left(M_{\infty}^{2} \sin ^{2} \beta_{o}-1\right)}-1\right]
$$

Note that this $\beta_{o}=\beta_{o}\left(\alpha, M_{\infty}\right)$ relationship defines a surface in 3-space of which the appropriate segment for the limited range of the present experiments is almost planar. By inspection, the $\beta_{o}-\alpha$ curves in this region are approximately parallel to each other and are offset from the $\alpha-M_{\infty}$ plane by the Mach angle $\mu_{\infty}$. Thus, subtracting $\mu_{\infty}$ from $\beta_{o}$ in effect "rotates" the $\beta_{o}$ surface so that the local region of interest is parallel to the

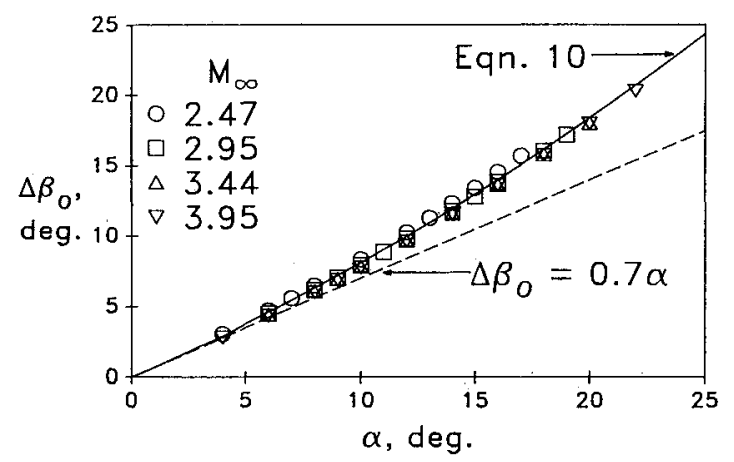

Fig. 5 Reduced shock angle vs fin angle of attack.

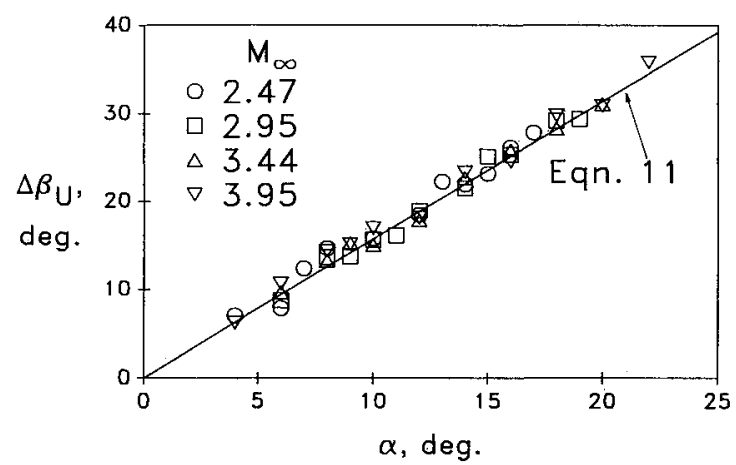

Fig. 6 Reduced upstream influence angle vs fin angle of attack. direction of view. This is shown in Fig. 5. A second-order least-square regression of $\mathrm{Eq}$. (9) for $2.5<M_{\infty}<4$ and $0<\alpha<22$ deg yields

$$
\Delta \beta_{o}=0.7 \alpha+0.011 \alpha^{2}
$$

Equation (10) conveniently expresses the fact that $\beta_{o}$, referenced to $\mu_{\infty}$, grows nonlinearly with $\alpha$ in the region of present interest.

The upstream influence angle response function $\Delta \beta_{U}$, plotted as a function of $\alpha$, is shown in Fig. 6. According to the present experiments, it can be observed that $\Delta \beta_{U}$ grows linearity with $\alpha$. Except for a small $M_{\infty}$ effect, the data approximately follow

$$
\Delta \beta_{U}=1.57 \alpha
$$

The physical reason for this linear behavior of $\Delta \beta_{U}$ with $\alpha$, as compared to the nonlinear behavior of $\Delta \beta_{o}$ with $\alpha$, is not entirely clear at present. It can be noted, however, that even for separated interactions, the upstream influence line marks the upstream extent of a compression-wave system in the interaction that coalesces into the forward leg of the familiar "lambda-foot" shock structure. This compression is always expected to be weak compared to the inviscid shock because the flow deflection away from the test surface for separated interactions is limited to $8-12 \mathrm{deg}$. If sufficiently weak, this compression at the upstream limit of the interaction might be modeled by a linearized theoretical description. Though this has not been done in the present study, it lends some physical significance to the observed linearity of $\Delta \beta_{U}$ with $\alpha$.

The linear growth of $\Delta \beta_{U}$ with $\alpha$ and the nonlinear growth of $\Delta \beta_{o}$ with $\alpha$, together, dictate $\Delta \beta_{U}$ and $\Delta \beta_{o}$ cannot be linearly related. This is illustrated in a plot of $\Delta \beta_{U}$ vs $\Delta \beta_{o}$ in Fig. 7. This figure shows that $\Delta \beta_{U}$ depends primarily on $\Delta \beta_{o}$ with a very weak secondary $M_{\infty}$ dependence, confirming the results of the dimensional analysis obtained earlier. Thus, to a good approximation, Eq. (8) can be simplified to

$$
\Delta \beta_{U}=f_{U}\left(\Delta \beta_{o}\right)
$$

over the present range. Figure 7 also shows that, for weak interactions, $\Delta \beta_{U}$ is more than twice as large as $\Delta \beta_{o}$, although the growth rate of $\Delta \beta_{U}$ decreases with increasing $\Delta \beta_{o}$.

Data scatter in earlier experiments at a single Mach number did not clearly reveal the behavior shown in Fig. 7. Many investigators, including the present authors, ${ }^{2,8-10}$ modeled the upstream influence behavior linearly by Eq. (3). The nonzero intercept $k_{2}$ was rationalized through arguments claiming rapid interaction growth for very weak shocks. The present results show that the assumption of a linear $\beta_{U}$ growth with $\beta_{o}$ is nonphysical. Such an assumption is only approximately valid for a limited range of $\beta_{o}$, as seen in Fig. 7, failing at both the weak and strong limits of shock strength. To replace this faulty assumption, from a second-order curvefit to the data, it

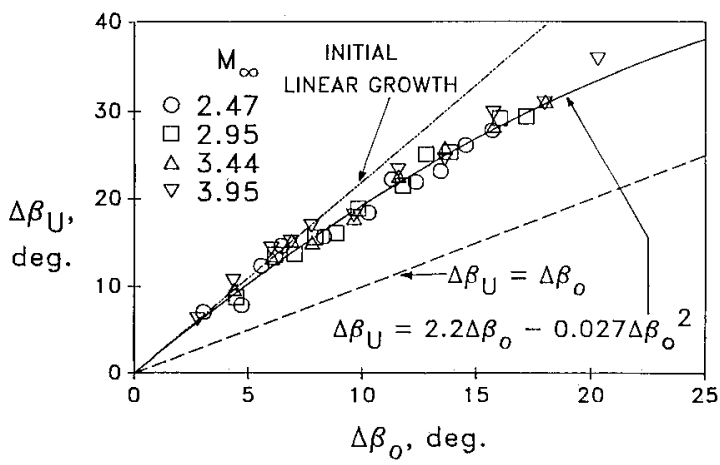

Fig. 7 Scaling of reduced upstream influence and shock angles. 


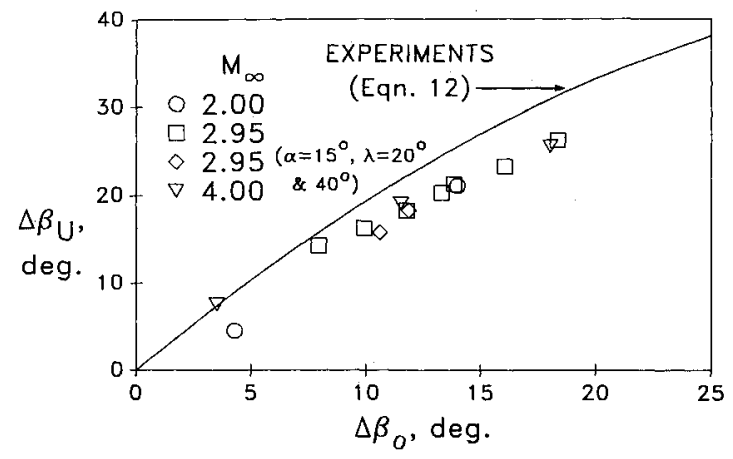

Fig. 8 Scaling of computed upstream influence and shock angles.

is proposed that

$$
\Delta \beta_{U}=\kappa_{1} \Delta \beta_{o}-\kappa_{2} \Delta \beta_{o}^{2}
$$

where $\kappa_{1} \approx 2.2$ and $\kappa_{2} \approx 0.027$. This equation demonstrates the nonlinear growth of $\beta_{U}$ with $\beta_{o}$ and accounts for variable Mach number (to first order) by referring both $\beta_{U}$ and $\beta_{o}$ to $\mu_{\infty}$. [Note that no particular physical significance is given to the polynomial form of Eq. (12) and that a trigonometric or other dependence would serve as well.]

Equation (12) cannot be extrapolated beyond the present experimental range, since doing so would eventually result in $\beta_{U}$ being less than $\beta_{o}$, a physically impossible situation. It is, however, hypothesized that in the extreme case of every strong interactions, $\beta_{U}$ will approach $\beta_{o}$. This limit is also shown in Fig. 7.

\section{Comparison with Computations}

As for the present computational solutions for $2<M_{\infty}<4$, Fig. 8 shows that although the computed $\Delta \beta_{U}$ correlates with $\Delta \beta_{o}$, the computed results fall below the experimental data. This implies that the computations underpredict the far-field interaction extent. Figure 8 also reveals that the computed results for weak interactions generated by $\alpha=5 \mathrm{deg}$ fins are within the experimental data scatter. However, for large $\alpha$, in which the separated region is also large, the computations are less successful in determining the interaction extent. This can also be seen in a comparison between the experimental surfaceflow visualization results (Fig. 9a) and the computed surfacefriction lines (Fig. 9b). It is obvious from these figures that the computed $\beta_{U}$ is smaller than the measured value.

Reasons for this discrepancy, considering both the grid resolution and turbulence model of the computation, have recently been discussed by Horstman. ${ }^{22}$ Briefly, a significant mesh refinement fails to increase the computed upstream influence of the interaction significantly. However, the use of a nonlinear turbulence model does increase the upstream influence slightly, though not enough to bring it into agreement with the present experiments. The complete explanation of this persistent underprediction remains unknown and is the subject of continued study.

\section{Other Correlating Parameters}

There is evidence prior to this study that the upstream influence location is a strong function of shock strength, ${ }^{1}$ although this evidence is based on studies at one or two Mach numbers. The present study shows that to account for the Mach number, the appropriate shock-strength parameter must be directly dependent on $\alpha$ and $\mu_{\infty}$ with an otherwise negligible $M_{\infty}$ dependence. Are there other shock strength parameters that might also be suitable? The shock-wave pressure coefficient $C_{p} \equiv 2\left(p_{r}-1\right) / \gamma M_{\infty}^{2}$ is one such candidate. However, $C_{p}$ has a Mach number dependence in that $C_{p} \equiv f\left(\alpha, M_{\infty}\right)$, so it is not a suitable parameter. Other parameters that do show the desired behavior with respect to $\alpha$ and $M_{\infty}$ are, for example,

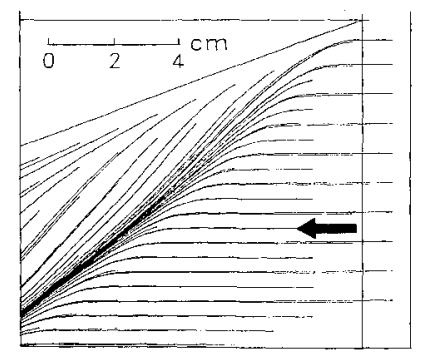

a)

Fig. 9 Comparison between experimental and computed skinfriction lines at $M_{\infty}=4, \alpha=20$ deg: a) experiment; b) computation.
$\left(M_{n}-1\right) / M_{\infty},\left(p_{r}-1\right) / M_{\infty}$, and $\Lambda \equiv\left(M_{\infty} / M_{2}-1\right)$, a parameter used successfully in two-dimensional impinging shock interactions. ${ }^{23}$ The reason these parameters can be used is, once again, that they are weak functions of $M_{\infty}$ (at least for the Mach number range of the present study).

\section{Final Remarks}

The present study shows that the Mach number effect on $\beta_{U}$ is simply accounted for by referencing $\beta_{U}$ and $\beta_{o}$ to the Mach angle. Physically, this result stems from the nature of the $\beta_{o}(\alpha$, $M_{\infty}$ ) relationship, wherein the only effect of $M_{\infty}$ enters through $\mu_{\infty}$ for the present range of variables. The residual $M_{\infty}$ trend of the data in Fig. 7 is extremely weak, such that it is within the overall data scatter. It may be concluded that the upstream influence response to compressibility effects on the turbulent boundary layer for $2.5 \leq M_{\infty} \leq 4$ and adiabatic-wall conditions is of second-order importance and thus may be neglected.

An examination of Fig. 7 reveals that a linearized theoretical approach to the upstream influence problem might be feasible for weak interactions., However, significant deviation from the initial linear growth occurs for $\alpha>5 \mathrm{deg}$. At the other extreme, for very strong shocks, $\beta_{U}$ is expected to approach $\beta_{o}$. The present study, therefore, also answers some of the questions raised by Wang and Bogdonoff, ${ }^{24}$ Bogdonoff and Wang, ${ }^{25}$ and Stalker $^{26}$ on whether these interactions are fundamentally conical or cylindrical. This answer is that, over a wide range of $\Delta \beta_{o}$, the interactions are conical. For small values of $\Delta \beta_{o}$, which result in small values of $\Delta \beta_{U}$, data scatter may give the impression of cylindrical symmetry. Unfortunately, experimental limitations prevent the present investigation and all known prior studies from observing this limit.

\section{Conclusions}

The present study shows that the Mach number effect on the far-field upstream influence line of fin-generated interactions can be simply accounted for by referencing $\beta_{U}$ and $\beta_{o}$ to $\mu_{\infty}$. It further reveals that a suitable shock-strength parameter for scaling $\Delta \beta_{U}$ is a function of $\alpha$ and not explicitly of $M_{\infty}$. The experimental results indicate that compressibility effects on the turbulent boundary layer are of secondary importance. Computations in the present Mach number range agree qualitatively with the experiments, especially in that far-field conical symmetry of surface features is shown. However, the computations underpredict the angular extent of these features at higher Mach numbers and shock strengths.

\section{Acknowledgments}

This research was supported by Grant NCA2-1R589-502 from NASA Ames Research Center, monitored by Dr. C. C. Horstman, and by Grant 86-0092 from the U. S. Air Force Office of Scientific Research, monitored by Dr. J. D. Wilson. The authors acknowledge the assistance of Mr. P. J. Barnhart in carrying out some of the experiments. 


\section{References}

${ }^{1}$ Settles, G. S. and Dolling, D. S., "Swept Shock Wave/BoundaryLayer Interactions," Progress in Astronautics and Aeronautics: Tactical Missile Aerodynamics, Vol. 104, edited by M. Hemsch and J. Nielsen, AIAA, New York, 1986, pp. 297-379.

${ }^{2}$ Settles, G. S. and Lu, F. K., "Conical Similarity of Shock/ Boundary Layer Interactions Generated by Swept and Unswept Fins," AIAA Journal, Vol. 23, July 1985, pp. 1021-1027.

${ }^{3}$ Inger, G. R., "Spanwise Propagation of Upstream Influence in Conical Swept Shock Boundary-Layer Interactions," AIAA Journal, Vol. 25, Feb. 1987, pp. 287-293.

${ }^{4}$ Hayes, J. R., "Prediction Techniques for the Characteristics of Fin-Generated Three Dimensional Shock Wave Turbulent Boundary Layer Interactions,' USAF AFDL-TR-77-10, May 1977.

5Zubin, M. A. and Ostapenko, N. A., "Structure of Flow in the Separation Region Resulting from Interaction of a Normal Shock Wave with a Boundary Layer in a Corner," Izvestiya Akademii Nauk SSSR, Mekhanika Zhidkosti i Gaza, Vol. 14, May-June 1979, pp. 51-58.

${ }^{6}$ Dolling, D. S. and Bogdonoff, S. M., "Upstream Influence in Sharp Fin-Induced Shock Wave/Turbulent Boundary-Layer Interaction," AIAA Journal, Vol. 21, Jan. 1983, pp. 143-145.

${ }^{7}$ Settles, G. S., "On the Inception Lengths of Swept Shock-Wave/ Turbulent Boundary-Layer Interactions," Proceedings of the IUTAM Symposium on Turbulent Shear-Layer/Shock-Wave Interactions, edited by J. Délery, Springer-Verlag, Berlin, 1986, pp. 203-213.

${ }^{8}$ Settles, G. S. and Kimmel, R. L., "Similarity of Quasiconical Shock Wave/Turbulent Boundary-Layer Interactions," AIAA Journal, Vol. 24, Jan. 1986, pp. 47-53.

${ }^{9}$ Goodwin, S. P., "An Exploratory Investigation of Sharp Fin-Induced Shock Wave-Turbulent Boundary-Layer Interactions at High Shock Strengths," M.S.E. Thesis, Mechanical and Aerospace Engineering Department, Princeton University, Princeton, NJ, Rept. No. 1687-T, Nov. 1984.

${ }^{10}$ Dolling, D. S., "Upstream Influence in Conically Symmetric Flow," AIAA Journal, Vol. 23, June 1985, pp. 967-969.

${ }^{11}$ Horstman, C. C., "Computation of Sharp-Fin-Induced Shock Wave/Turbulent Boundary-Layer Interactions,' AIAA Journal, Vol. 24, Sept. 1986, pp. 1433-1440.

${ }^{12}$ Zheltovodov, A. A., "Properties of Two- and Three-Dimensional Separation Flows at Supersonic Velocities," Izvestiya Akademii Nauk SSSR, Mekhanika Zhidkosti i Gaza, Vol. 14, May-June 1979, pp. $42-50$.

${ }^{13}$ Zheltovodov, A. A., Pavlov, A. A., Shilein, E. H., and Yakovlev, V. N., "Interconnectionship Between the Flow Separation and the Direct and Inverse Transition at Supersonic Speed Conditions," Pro- ceedings of the IUTAM Symposium on Laminar-Turbulent Transition, edited by V. V. Kozlov, Springer-Verlag, Berlin, 1985, pp. 503-508.

${ }^{14}$ Zheltovodov, A. A., "Regimes and Properties of Three-Dimensional Separation Flows Initiated by Skewed Compression Shocks," Zhurnal Prikladnoi Mekhaniki i Tekhnicheskoi Fiziki, No. 3, May-June 1982, pp. 116-123.

${ }^{15}$ Lu, F. K., "Fin-Generated Shock-Wave Boundary-Layer Interactions," Ph.D. Thesis, Mechanical Engineering Dept., Pennsylvania State University, University Park, PA, Feb. 1988.

${ }^{16}$ McClure, W. B., "An Experimental Study into the Scaling of the Interaction of an Unswept Sharp Fin-Generated Shock/Turbulent Boundary Layer Interaction,"' M.S.E. Thesis, Princeton University, Princeton, NJ, Jan. 1983.

${ }^{17}$ Sun, C. C. and Childs, M. E., "A Modified Wall-Wake Velocity Profile for Turbulent Compressible Boundary Layers," Journal of Aircraft, Vol. 10, June 1973, pp. 318-383.

${ }^{18}$ Settles, G. S. and Teng H.-Y., "Flow Visualization Methods for Separated Three-Dimensional Shock Wave/Turbulent Boundary Layer Interactions," AIAA Journal, Vol. 21, March 1983, pp. 390-397.

${ }^{19}$ Gramann, R. A. and Dolling, D. S., "Detection of Turbulen Boundary Layer Separation Using Fluctuating Wall Pressure Signals," AIAA Paper 88-4676, Sept. 1988.

${ }^{20}$ Knight, D. D., Horstman, C. C., Shapey, B., and Bogdonoff, S. M., "Structure of Supersonic Turbulent Flow Past a Fin," $A I A A$ Journal, Vol. 25, Oct. 1987, pp. 1331-1337.

${ }^{21}$ Korkegi, R. H., "A Simple Correlation for Incipient Turbulent Boundary-Layer Separation due to a Skewed Shock Wave," $A I A A$ Journal, Nov. 1973, pp. 1578-1579.

${ }^{22}$ Horstman, C. C., "Prediction of Secondary Separation in Shock Wave Boundary-Layer Interactions," Computers and Fluids (submitted for publication), July 1988.

${ }^{23}$ Green, J. E., "Interactions Between Shock Waves and Turbulent Boundary Layers," Progress in Aerospace Sciences, Vol. 11, edited by D. Küchmann, Pergamon, Oxford, U. K., 1970, pp. 235-340.

${ }^{24}$ Wang, S.-Y., and Bogdonoff, S. M., "A Re-Examination of the Upstream Influence Scaling and Similarity Laws for 3-D Shock Wave/ Turbulent Boundary-Layer Interaction," AIAA Paper 86-0347, Jan. 1986.

${ }^{25}$ Bogdonoff, S. M. and Wang, S.-Y., "Comment on 'Conical Similarity of Shock/Boundary Layer Interactions Generated by Swept and Unswept Fins',' AIAA Journal, Vol. 24, March 1986, p. 540.

${ }^{26}$ Stalker, R. J., "A Characteristics Approach to Swept Shock Wave Boundary Layer Interactions," AIAA Journal, Vol. 22, Nov. 1984, pp. 1626-1632. 